\title{
An alternative functional equation of Jensen type on groups
}

\author{
Choodech Srisawat, Nataphan Kitisin*, Paisan Nakmahachalasint \\ Department of Mathematics and Computer Science, Faculty of Science, Chulalongkorn University, \\ Bangkok 10330 Thailand
}

*Corresponding author, e-mail: nataphan.k@chula.ac.th

Received 19 Feb 2015

Accepted 19 Aug 2015

\begin{abstract}
Given an integer $\lambda \neq 2$, we establish the general solution of an alternative functional equation of Jensen type on certain groups. First, we give a criterion for the existence of the general solution for the functional equation $f\left(x y^{-1}\right)-2 f(x)+f(x y)=0$ or $f\left(x y^{-1}\right)-\lambda f(x)+f(x y)=0$, where $f$ is a mapping from a group $(G, \cdot)$ to a uniquely divisible abelian group $(H,+)$. Then we show that, for $\lambda \notin\{0,-1,-2\}$, the above alternative functional equation is equivalent to the classical Jensen's functional equation. We also find the general solution in the case when $G$ is a cyclic group and $\lambda \neq 2$ is an integer.
\end{abstract}

KEYWORDS: Jensen's functional equation, additive function

MSC2010: 39B52 39B05

\section{INTRODUCTION}

The alternative functional equation has been widely investigated. For instance, Kannappan and Kuczma ${ }^{1}$ studied the solutions of the alternative Cauchy functional equation

$$
\begin{aligned}
& (f(x+y)-a f(x)-b f(y)) \\
& \quad(f(x+y)-f(x)-f(y))=0
\end{aligned}
$$

on an abelian group. $\mathrm{Ger}^{2}$ extended the results in Ref. 1 with the same domain to the alternative functional equation

$$
\begin{aligned}
& (f(x+y)-a f(x)-b f(y)) \\
& (f(x+y)-c f(x)-d f(y))=0 .
\end{aligned}
$$

Kuczma $^{3}$ proved that the alternative Cauchy functional equation (1) on a semigroup is equivalent to the classical Cauchy functional equation in the case when $a=b=-1$. Forti ${ }^{4}$ then successfully established the general solution of (1) in a more general setting, finding the general solution to the alternative functional equation

$$
\begin{aligned}
(c f(x+y)-a f(x)-b f(y)-d) & \\
( & (f(x+y)-f(x)-f(y))=0
\end{aligned}
$$

on an abelian group by extending the work of Kannappan and Kuczma ${ }^{1}$.
Motivated by the work on the alternative Cauchy functional equation and many extensive studies on Jensen's functional equation on different kinds of groups (see, e.g., Refs. 5-9) Nakmahachalasint ${ }^{10}$ has investigated the alternative Jensen's functional equation of the form

$$
f(x) \pm 2 f(x y)+f\left(x y^{2}\right)=0
$$

on a semigroup. His work is a significant generalization of the work in Refs. 11, 12 on the classical Jensen's functional equation on a group. Note that one of the results in Ref. 10 is that if the domain of the alternative Jensen's functional equation (2) is a 2-divisible group, then (2) is equivalent to the classical Jensen's functional equation in the sense that the solution $f$ satisfies Jensen's functional equation

$$
f(x)-2 f(x y)+f\left(x y^{2}\right)=0,
$$

for all $x, y$ in the domain.

Inspired by the work of Nakmahachalasint ${ }^{10}$ and Forti $^{4}$, we will investigate the alternative Jensen's functional equation in an even more general setting. Namely, given an integer $\lambda \neq 2$, we find a criterion of the existence of the general solution of the alternative Jensen's functional equation

$$
\begin{aligned}
& f\left(x y^{-1}\right)-2 f(x)+f(x y)=0 \quad \text { or } \\
& f\left(x y^{-1}\right)-\lambda f(x)+f(x y)=0,
\end{aligned}
$$


where $f$ is a mapping from a group $(G, \cdot)$ to a uniquely divisible abelian group $(H,+)$. Note that when $\lambda=-2$, we recover the results in Ref. 10 in the case when the domain is a group. Furthermore, if $\lambda \notin\{0,-1,-2\}$, then we show that (3) is equivalent to the classical Jensen's functional equation

$$
f\left(x y^{-1}\right)-2 f(x)+f(x y)=0 .
$$

We also give the general solution in the case when the domain is a cyclic group.

\section{NOTATION AND DEFINITIONS}

Throughout the paper, we let $(G, \cdot)$ be a group and let $(H,+)$ be a uniquely divisible abelian group. Recall that $(H,+)$ is uniquely divisible provided that $(H,+)$ has the following properties.

(i) For all $a \in H$ and $n \in \mathbb{Z}^{+}$, there exists $b \in H$ such that $n b=a$.

(ii) For all $a, b \in H$ and $n \in \mathbb{Z}^{+}$, if $n a=n b$, then $a=b$.

We introduce the following notation for sequences $\left(a_{k}\right)_{k \in \mathbb{Z}}$ in $H$. We say that $\left(a_{k}\right)_{k \in \mathbb{Z}}=(\bar{\alpha}, \bar{\beta})$ when there exists $k_{0} \in \mathbb{Z}$ with $a_{i}=\alpha$ for all $i<k_{0}$ and $a_{\mathrm{j}}=\beta$ for all $i \geqslant k_{0}$, i.e.,

$$
(\ldots, \alpha, \alpha, \quad \beta, \beta, \ldots)=(\bar{\alpha}, \bar{\beta}) .
$$

We say that $\left(a_{k}\right)_{k \in \mathbb{Z}}=(\bar{\alpha}, \beta, \bar{\gamma})$ when there exists $k_{0} \in \mathbb{Z}$ with $a_{i}=\alpha$ for all $i<k_{0}, a_{k_{0}}=\beta$, and $a_{i}=\gamma$ for all $i>k_{0}$, i.e.,

$$
(\ldots, \alpha, \alpha, \quad \beta, \quad \gamma, \gamma, \ldots)=(\bar{\alpha}, \beta, \bar{\gamma}) .
$$

Let $p$ be a positive integer. We say that $\left(a_{k}\right)_{k \in \mathbb{Z}}=$ $\left(\overline{\alpha_{0}, \ldots, \alpha_{p-1}}\right)$ when there exists $k_{0} \in \mathbb{Z}$ such that $a_{i}=\alpha_{k_{0}+i(\bmod p)}$ for all $i \in \mathbb{Z}$. In other words, $\left(\overline{\alpha_{0}, \ldots, \alpha_{p-1}}\right)$ is a periodic sequence of a period $p$, i.e.,

$$
\begin{array}{r}
\left(\ldots, \quad \alpha_{0}, \ldots, \alpha_{p-1}, \quad \alpha_{0}, \ldots, \alpha_{p-1}, \ldots\right) \\
=\left(\overline{\alpha_{0}, \ldots, \alpha_{p-1}}\right) .
\end{array}
$$

Given an integer $\lambda$ and a function $f: G \rightarrow H$, for every pair of $x, y \in G$, we define

$$
F_{y}^{(\lambda)}(x):=f\left(x y^{-1}\right)-\lambda f(x)+f(x y) .
$$

For $\lambda \neq 2$, we write

$$
\mathscr{P} f_{y}^{(\lambda)}(x):=\left(F_{y}^{(2)}(x)=0 \quad \text { or } \quad F_{y}^{(\lambda)}(x)=0\right) .
$$

Let $\mathscr{A}_{(G, H)}^{(\lambda)}$ be the set of all solutions of (3), i.e., $\mathscr{A}_{(G, H)}^{(\lambda)}:=\left\{f: G \rightarrow H \mid \mathscr{P} f_{y}^{(\lambda)}(x)\right.$ for all $\left.x, y \in G\right\}$, whereas the set of solutions of Jensen's functional equation will be denoted by

$\mathscr{J}_{(G, H)}:=\left\{f: G \rightarrow H \mid F_{y}^{(2)}(x)=0\right.$ for all $\left.x, y \in G\right\}$.

Remark 1 Let $f \in \mathscr{A}_{(G, H)}^{(\lambda)}$. Given $x \in G$, it should be noted that if $f(x)=0$, then both alternatives in $\mathscr{P} f_{y}^{(\lambda)}(x)$ are equivalent, and we can infer that $F_{y}^{(2)}(x)=0$ and $F_{y}^{(\lambda)}(x)=0$ for all $y \in G$. However, if $f(x) \neq 0$, then for each $y \in G$, only one alternative in $\mathscr{P} f_{y}^{(\lambda)}(x)$ will hold.

The above remark is an important fact that will be used extensively later on and should be kept in mind when reading the proofs below.

\section{FUNDAMENTAL LEMMAS}

We will now prove some fundamental lemmas concerning the relation between $\mathscr{P} f_{y}^{(\lambda)}\left(x y^{-1}\right)$, $\mathscr{P} f_{y}^{(\lambda)}(x)$, and $\mathscr{P} f_{y}^{(\lambda)}(x y)$.

Lemma 1 Let $f \in \mathscr{A}_{(G, H)}^{(\lambda)}$ and let $x, y \in G$.

(i) If $F_{y}^{(2)}\left(x y^{-1}\right)=0$ and $F_{y}^{(2)}(x y)=0$, then $F_{y}^{(2)}(x)=0$.

(ii) If $F_{y}^{(\lambda)}\left(x y^{-1}\right)=0$ and $F_{y}^{(\lambda)}(x y)=0$, then $F_{y}^{(\lambda)}(x)=0$.

Proof: Suppose that all the assumptions in the lemma hold.

(i) If $F_{y}^{(2)}(x) \neq 0$, then $F_{y}^{(\lambda)}(x)=0$. Hence $F_{y}^{(2)}\left(x y^{-1}\right)+2 F_{y}^{(\lambda)}(x)+F_{y}^{(2)}(x y)=0$, i.e,

$$
f\left(x y^{-2}\right)+2(1-\lambda) f(x)+f\left(x y^{2}\right)=0 .
$$

Consider $\mathscr{P} f_{y^{2}}^{(\lambda)}(x)$. The alternative

$$
f\left(x y^{-2}\right)-2 f(x)+f\left(x y^{2}\right)=0
$$

and (5) gives $(2-\lambda) f(x)=0$, while the alternative

$$
f\left(x y^{-2}\right)-\lambda f(x)+f\left(x y^{2}\right)=0
$$

and (5) also gives $(2-\lambda) f(x)=0$. Since $\lambda \neq 2$, we get $f(x)=0$. Hence $F_{y}^{(2)}(x)=0$ by Remark 1 .

(ii) If $F_{y}^{(\lambda)}(x) \neq 0$, then $F_{y}^{(2)}(x)=0$. Hence $F_{y}^{(\lambda)}\left(x y^{-1}\right)+\lambda F_{y}^{(2)}(x y)+F_{y}^{(\lambda)}(x)=0$, i.e.,

$$
f\left(x y^{-2}\right)+2(1-\lambda) f(x)+f\left(x y^{2}\right)=0 .
$$

By a similar argument to the above, we will have $f(x)=0$. Hence $F_{y}^{(\lambda)}(x)=0$ by Remark 1 . 
Lemma 2 Let $f \in \mathscr{A}_{(G, H)}^{(\lambda)}$ and let $x, y \in G$. If $F_{y}^{(2)}\left(x y^{-1}\right)=0, F_{y}^{(2)}(x) \neq 0$ and $F_{y}^{(\lambda)}\left(x y^{2}\right) \neq 0$, then $\lambda=0$ and

$$
f\left(x y^{n}\right)= \begin{cases}-f(x), & \text { if } n \in \mathbb{Z}^{+}, \\ f(x), & \text { if } n \in \mathbb{Z}^{-} .\end{cases}
$$

Proof: Suppose that all the assumptions in the lemma hold. By Remark 1, $F_{y}^{(2)}(x) \neq 0$ implies that $f(x) \neq 0$. From $F_{y}^{(2)}(x) \neq 0$ and $\mathscr{P} f_{y}^{(\lambda)}(x)$, we obtain $F_{y}^{(\lambda)}(x)=0$. From $F_{y}^{(\lambda)}\left(x y^{2}\right) \neq 0$ and $\mathscr{P} f_{y}^{(\lambda)}\left(x y^{2}\right)$, we get $F_{y}^{(2)}\left(x y^{2}\right)=0$. From $F_{y}^{(2)}\left(x y^{-1}\right)=0$ and $F_{y}^{(2)}(x) \neq 0$, Lemma 1 gives $F_{y}^{(2)}(x y) \neq 0$. By the alternatives in $\mathscr{P} f_{y}^{(\lambda)}(x y)$, we obtain $F_{y}^{(\lambda)}(x y)=0$. If $F_{y}^{(\lambda)}\left(x y^{3}\right)=0$, then by Lemma 1, we must have $F_{y}^{(\lambda)}\left(x y^{2}\right)=0$, a contradiction. Hence $F_{y}^{(\lambda)}\left(x y^{3}\right) \neq 0$ and the alternatives in $\mathscr{P} f_{y}^{(\lambda)}\left(x y^{3}\right)$ gives $F_{y}^{(2)}\left(x y^{3}\right)=0$.

Eliminating $f\left(x y^{-1}\right)$ from $F_{y}^{(2)}\left(x y^{-1}\right)=0$ and $F_{y}^{(\lambda)}(x)=0$, we get

$$
f\left(x y^{-2}\right)+(1-2 \lambda) f(x)+2 f(x y)=0 .
$$

We consider each alternative in $\mathscr{P} f_{y^{2}}^{(\lambda)}(x)$ as follows.

(i) Assume that $F_{y^{2}}^{(2)}(x)=0$. Solving $F_{y}^{(\lambda)}(x y)=0$, $F_{y^{2}}^{(2)}(x)=0$ and (6), we have

$$
\begin{aligned}
f(x y)+2 f(x) & =0 \text { and } \\
f\left(x y^{2}\right)+(1+2 \lambda) f(x) & =0 .
\end{aligned}
$$

From $F_{y}^{(2)}\left(x y^{2}\right)=0$ and (7), we obtain $f\left(x y^{3}\right)+$ $4 \lambda f(x)=0$. Considering the alternatives in $\mathscr{P} f_{y^{2}}^{(\lambda)}(x y)$, we conclude that $(\lambda-2) f(x)=$ 0 . Since $\lambda \neq 2$, we must have $f(x)=0$, a contradiction. Thus this case does not exist.

(ii) Assume that $F_{y^{2}}^{(\lambda)}(x)=0$. Solving $F_{y}^{(\lambda)}(x y)=0$, $F_{y^{2}}^{(\lambda)}(x)=0$ and (6), we have

$$
\begin{aligned}
f(x)+f(x y) & =0 \text { and } \\
f\left(x y^{2}\right)+(1+\lambda) f(x) & =0 .
\end{aligned}
$$

Eliminating $f\left(x y^{2}\right)$ and $f\left(x y^{3}\right)$ from $F_{y}^{(2)}\left(x y^{2}\right)=0, \quad F_{y}^{(2)}\left(x y^{3}\right)=0$ and (8), we get

$$
f\left(x y^{4}\right)+(1+3 \lambda) f(x)=0 .
$$

We will consider the alternative in $\mathscr{P} f_{y^{2}}^{(\lambda)}\left(x y^{2}\right)$ as follows. If $F_{y^{2}}^{(2)}\left(x y^{2}\right)=0$, then from (8),
(9) and $F_{y^{2}}^{(2)}\left(x y^{2}\right)=0$, we conclude that $(\lambda-$ 2) $f(x)=0$. Since $\lambda \neq 2$, we have $f(x)=0$, a contradiction. Thus we must get $F_{y^{2}}^{(\lambda)}\left(x y^{2}\right)=0$. Solving (8), (9) and $F_{y^{2}}^{(\lambda)}\left(x y^{2}\right)=0$, we conclude that $(\lambda-2) \lambda f(x)=0$. Since $\lambda \neq 2$ and $f(x) \neq$ 0 , we must have $\lambda=0$ and so $F_{y^{2}}^{(0)}(x)=0$.

We already have $\lambda=0$. From (8), (9) and $F_{y}^{(2)}\left(x y^{2}\right)=0$, we obtain

$$
f\left(x y^{n}\right)=-f(x) \text { for all } n=1, \ldots, 4 .
$$

We will prove that (10) also holds for all $n \in \mathbb{Z}^{+}$. It is only left to prove that $F_{y}^{(2)}\left(x y^{n}\right)=0$ for all $n \geqslant 4$. We will show this by contradiction. Suppose that $m \geqslant 4$ is the smallest integer satisfying $F_{y}^{(2)}\left(x y^{m}\right) \neq$ 0 . Thus the alternatives in $\mathscr{P} f_{y}^{(0)}\left(x y^{i}\right)$ for each $n=$ $3, \ldots, m-1$ give $F_{y}^{(2)}\left(x y^{i}\right)=0$, i.e.,

$$
f\left(x y^{i-1}\right)-2 f\left(x y^{i}\right)+f\left(x y^{i+1}\right)=0
$$

for all $n=3, \ldots, m-1$. From (11) and $f\left(x y^{3}\right)=$ $f\left(x y^{4}\right)=-f(x)$ in (10), we have

$$
f\left(x y^{n}\right)=-f(x) \text { for all } n=1, \ldots, m .
$$

The alternatives in $\mathscr{P} f_{y}^{(0)}\left(x y^{m}\right)$ and $F_{y}^{(2)}\left(x y^{m}\right) \neq$ 0 gives $F_{y}^{(0)}\left(x y^{m}\right)=0$. By $F_{y}^{(0)}\left(x y^{m}\right)=0$ and $f\left(x y^{m-1}\right)=-f(x)$ in (12), we get $f\left(x y^{m+1}\right)=$ $f(x)$.

(i) If $m=2 k$ for some $k \geqslant 2$, then $f\left(x y^{2 k+1}\right)=$ $f\left(x y^{m+1}\right)=f(x) . \quad$ From $F_{y}^{(0)}(x)=0$ and $f(x y)=-f(x)$ in (12), we obtain $f\left(x y^{-1}\right)=$ $f(x)$. Since $2 \leqslant k<m, f\left(x y^{k}\right)=-f(x)$ by (12). The alternatives in $\mathscr{P} f_{y^{k+1}}^{(0)}\left(x y^{k}\right)$ gives $f(x)=0$, a contradiction.

(ii) If $m=2 k+1$ for some $k \geqslant 2$, then $f\left(x y^{2 k+2}\right)=f\left(x y^{m+1}\right)=f(x)$. Since $2 \leqslant k<m, f\left(x y^{k+1}\right)=-f(x)$ by (12). The alternatives in $\mathscr{P} f_{y^{k+1}}^{(0)}\left(x y^{k+1}\right)$ gives $f(x)=0$, a contradiction.

Thus $f\left(x y^{n}\right)=-f(x)$ for all $n \in \mathbb{Z}^{+}$, as desired.

Since $F_{y}^{(0)}(x)=0, F_{y^{2}}^{(0)}(x)=0$ and $f(x y)=$ $f\left(x y^{2}\right)=-f(x)$ in (10), we have $f\left(x y^{-2}\right)=$ $f\left(x y^{-1}\right)=f(x)$. We can repeat the above process to show that $f\left(x y^{n}\right)=f(x)$ for all $n \in \mathbb{Z}^{-}$by replacing $x$ by $x y^{-2}$ and $y$ by $y^{-1}$ in the previous arguments.

Lemma 3 Let $f \in \mathscr{A}_{(G, H)}^{(\lambda)}$ and let $x, y \in G$.

(i) If $F_{y}^{(\lambda)}\left(x y^{-1}\right)=0$, and $F_{y}^{(2)}(x) \neq 0$ and $F_{y}^{(\lambda)}(x y)=0$, then $\lambda \in\{-1,-2\}$. 
(ii) If $F_{y}^{(\lambda)}\left(x y^{-1}\right) \neq 0$ and $F_{y}^{(2)}(x) \neq 0$, then $\lambda \neq-2$.

Proof: Suppose that all the assumptions in the lemma hold.

(i) From $F_{y}^{(2)}(x) \neq 0$ and $\mathscr{P} f_{y}^{(\lambda)}(x)$, we have $F_{y}^{(\lambda)}(x)=0$. Hence $f(x) \neq 0$ by Remark 1 . Observe that $F_{y}^{(\lambda)}\left(x y^{-1}\right)+\lambda F_{y}^{(\lambda)}(x)+F_{y}^{(\lambda)}(x y)=0$ reduces to

$$
f\left(x y^{-2}\right)+\left(2-\lambda^{2}\right) f(x)+f\left(x y^{2}\right)=0 .
$$

We will now consider each alternative in $\mathscr{P} f_{y^{2}}^{(\lambda)}(x)$. The alternative $f\left(x y^{-2}\right)-2 f(x)+f\left(x y^{2}\right)=0$ and (13) gives $\left(4-\lambda^{2}\right) f(x)=0$, while the alternative $f\left(x y^{-2}\right)-\lambda f(x)+f\left(x y^{2}\right)=0$ and (13) gives $(2+$ $\left.\lambda-\lambda^{2}\right) f(x)=0$. Since $f(x) \neq 0$ and $\lambda \neq 2$, we must have $\lambda \in\{-1,-2\}$.

(ii) We will prove $\lambda \neq-2$ by contradiction. Suppose that $\lambda=-2$. From $F_{y}^{(-2)}\left(x y^{-1}\right) \neq 0$ and $\mathscr{P} f_{y}^{(-2)}\left(x y^{-1}\right)$, we obtain $F_{y}^{(2)}\left(x y^{-1}\right)=0$. From $F_{y}^{(2)}(x) \neq 0$ and $\mathscr{P} f_{y}^{(-2)}(x)$, we get $F_{y}^{(-2)}(x)=0$ and thus $f(x) \neq 0$ by Remark 1 . Since $F_{y}^{(-2)}\left(x y^{-1}\right) \neq 0$ and $F_{y}^{(-2)}(x)=0$, Lemma 1 gives $F_{y}^{(-2)}\left(x y^{-2}\right) \neq$ 0 . Thus by the alternatives in $\mathscr{P} f_{y}^{(-2)}\left(x y^{-2}\right)$, we have $F_{y}^{(2)}\left(x y^{-2}\right)=0$. From $F_{y}^{(2)}\left(x y^{-1}\right)=0$ and $F_{y}^{(2)}(x) \neq 0$, Lemma 1 gives $F_{y}^{(2)}(x y) \neq 0$. By the alternatives in $\mathscr{P} f_{y}^{(-2)}(x y)$, we obtain $F_{y}^{(-2)}(x y)=$ 0 . Eliminating $f\left(x y^{-1}\right)$ from $F_{y}^{(2)}\left(x y^{-1}\right)=0$ and $F_{y}^{(-2)}(x)=0$, we get

$$
f\left(x y^{-2}\right)+5 f(x)+2 f(x y)=0 .
$$

Eliminating $f\left(x y^{-2}\right)$ and $f\left(x y^{2}\right)$ from $F_{y}^{(-2)}(x y)=$ 0 , (14) and each alternative in $\mathscr{P} f_{y^{2}}^{(2)}(x)$, we obtain

$$
2 f(x)+f(x y)=0 \text { or } f(x)+f(x y)=0 .
$$

From $F_{y}^{(2)}\left(x y^{-2}\right)=0, \quad F_{y}^{(2)}\left(x y^{-1}\right)=0 \quad$ and $F_{y}^{(-2)}(x)=0$, we have $F_{y}^{(2)}\left(x y^{-2}\right)+2 F_{y}^{(2)}\left(x y^{-1}\right)+$ $3 F_{y}^{(-2)}(x)=0$, i.e.,

$$
f\left(x y^{-3}\right)+8 f(x)+3 f(x y)=0 .
$$

Eliminating $f\left(x y^{-3}\right)$ from (16) and each alternatives in $\mathscr{P} f_{y^{2}}^{(-2)}\left(x y^{-1}\right)$, we get

$$
\begin{aligned}
& 4 f(x)+f(x y)+f\left(x y^{-1}\right)=0 \text { or } \\
& 4 f(x)+f(x y)-f\left(x y^{-1}\right)=0 .
\end{aligned}
$$

Solving $F_{y}^{(-2)}(x)=0$ and (17), we conclude that

$$
f(x)=0 \text { or } 3 f(x)+f(x y)=0 .
$$

Combining (15) and (18), we have $f(x)=0$, a contradiction. Hence we must get $\lambda \neq-2$, as desired.

Lemma 4 Let $f \in \mathscr{A}_{(G, H)}^{(\lambda)}$ and let $x, y \in G$. If $F_{y}^{(\lambda)}\left(x y^{-1}\right) \neq 0, F_{y}^{(2)}(x) \neq 0$ and $F_{y}^{(\lambda)}\left(x y^{2}\right)=0$, then $\lambda=-1$ and

(i) $\left(f\left(x y^{n}\right)\right)_{n \in \mathbb{Z}}=(\bar{a},-2 a, \bar{a})$ for some $a \in H$, or

(ii) $\left(f\left(x y^{n}\right)\right)_{n \in \mathbb{Z}}=(\overline{-2 a, a, \ldots, a})$, a periodic sequence of an odd period $p \geqslant 5$, for some $a \in H$.

Proof: Suppose that all the assumptions in the lemma hold. Thus $\lambda \neq-2$ by Lemma 3 . From $F_{y}^{(\lambda)}\left(x y^{-1}\right) \neq 0$ and $\mathscr{P} f_{y}^{(\lambda)}\left(x y^{-1}\right)$, we get $F_{y}^{(2)}\left(x y^{-1}\right)=0$. From $F_{y}^{(2)}(x) \neq 0$ and $\mathscr{P} f_{y}^{(\lambda)}(x)$, we have $F_{y}^{(\lambda)}(x)=0$. Hence $f\left(x y^{-1}\right) \neq 0$ and $f(x) \neq 0$ by Remark 1 . Since $F_{y}^{(2)}(x) \neq 0$ and $F_{y}^{(2)}\left(x y^{-1}\right)=0$, Lemma 1 gives $F_{y}^{(2)}(x y) \neq 0$. By the alternatives in $\mathscr{P} f_{y}^{(\lambda)}(x y)$, we get $F_{y}^{(\lambda)}(x y)=$ 0 . From $F_{y}^{(\lambda)}(x)=0, F_{y}^{(2)}(x y) \neq 0, F_{y}^{(\lambda)}\left(x y^{2}\right)=0$ and $\lambda \neq-2$, Lemma 3 gives $\lambda=-1$. Eliminating $f\left(x y^{-1}\right)$ from $F_{y}^{(2)}\left(x y^{-1}\right)=0$ and $F_{y}^{(-1)}(x)=0$, we obtain

$$
f\left(x y^{-2}\right)+3 f(x)+2 f(x y)=0 .
$$

Consider the alternatives in $\mathscr{P} f_{y^{2}}^{(-1)}(x)$ as follows. Solving $F_{y^{2}}^{(2)}(x)=0, F_{y}^{(-1)}(x y)=0$ and (19) gives $2 f(x)+f(x y)=0$, while solving $F_{y^{2}}^{(-1)}(x)=0$, $F_{y}^{(-1)}(x y)=0$ and (19) gives $f(x)+f(x y)=0$. If $f(x)+f(x y)=0$, then $F_{y}^{(-1)}(x)=0$ simplifies to $f\left(x y^{-1}\right)=0$, a contradiction. Thus we must have $2 f(x)+f(x y)=0$. Let $f(x)=a$. From $F_{y}^{(2)}\left(x y^{-1}\right)=0, F_{y}^{(-1)}(x)=0, F_{y}^{(-1)}(x y)=0$, $F_{y}^{(-1)}\left(x y^{2}\right)=0$ and $2 f(x)+f(x y)=0$, we conclude that

$$
\begin{array}{r}
\left(f\left(x y^{-2}\right), f\left(x y^{-1}\right), f(x), f(x y), f\left(x y^{2}\right), f\left(x y^{3}\right)\right) \\
=(a, a, a,-2 a, a, a) .
\end{array}
$$

If $F_{y}^{(-1)}\left(x y^{3}\right)=0$, then by $f\left(x y^{2}\right)=f\left(x y^{3}\right)=a$ in (20), we get $f\left(x y^{4}\right)=-2 a$. From $f\left(x y^{-2}\right)=$ $a$ and $f(x y)=-2 a$ in (20), the alternatives in $\mathscr{P} f_{y^{3}}^{(-1)}(x y)$ gives $a=0$, a contradiction. Hence $F_{y}^{(-1)}\left(x y^{3}\right) \neq 0$. By the alternatives in $\mathscr{P} f_{y}^{(-1)}\left(x y^{3}\right)$, we have $F_{y}^{(2)}\left(x y^{3}\right)=0$, i.e., $f\left(x y^{4}\right)=a$. From the alternatives in $\mathscr{P} f_{y}^{(-1)}\left(x y^{n}\right)$ for each $n \geqslant 4$, we consider two possible cases as follows. 
(i) Assume that $F_{y}^{(2)}\left(x y^{n}\right)=0$ for all $n \geqslant 4$, i.e.,

$$
f\left(x y^{n-1}\right)-2 f\left(x y^{n}\right)+f\left(x y^{n+1}\right)=0
$$

for all $n \geqslant 4$. From (21), $f\left(x y^{4}\right)=a$ and $f\left(x y^{2}\right)=$ $f\left(x y^{3}\right)=a$ in (20), we conclude that

$$
f\left(x y^{n}\right)=a \text { for all } n \geqslant 2 .
$$

Next, we show that (22) also holds for all $n \leqslant 0$. It is only left to prove that $f\left(x y^{n}\right)=a$ for all $n \leqslant-3$. Let $m \leqslant-3$ be an integer. We consider the alternatives in $\mathscr{P} f_{y^{m-1}}^{(-1)}(x y)$ as follows. From $f(x y)=$ $-2 a$ in (20) and $f\left(x y^{-m+2}\right)=a$ in (22), the alternative $F_{y^{m-1}}^{(-1)}(x y)=0$ and (22) gives $f\left(x y^{m}\right)=$ $-5 a$, while the alternative $F_{y^{m-1}}^{(-1)}(x y)=0$ and (22) gives $f\left(x y^{m}\right)=a$. First, assume that $f\left(x y^{m}\right)=$ $-5 a$. From $f(x)=a$ in (20) and $f\left(x y^{-m}\right)=a$ in (22), the alternatives in $\mathscr{P} f_{y^{m}}^{(-1)}(x)$ give $a=0$, a contradiction. Thus we must have $f\left(x y^{m}\right)=a$. Hence we get that (22) holds for all $n \leqslant 0$ and so $\left(f\left(x y^{n}\right)\right)_{n \in \mathbb{Z}}=(\bar{a},-2 a, \bar{a})$.

(ii) Assume that $m \geqslant 4$ is the smallest integer satisfying $F_{y}^{(2)}\left(x y^{m}\right) \neq 0$. From the alternatives in $\mathscr{P} f_{y}^{(-1)}\left(x y^{n}\right)$ for each $3 \leqslant n \leqslant m-1$, we have $F_{y}^{(2)}\left(x y^{n}\right)=0$, i.e.,

$$
f\left(x y^{n-1}\right)-2 f\left(x y^{n}\right)+f\left(x y^{n+1}\right)=0
$$

for all $3 \leqslant n \leqslant m-1$. Since (23) and $f\left(x y^{2}\right)=$ $f\left(x y^{3}\right)=a$ in (20), we obtain

$$
f\left(x y^{n}\right)=a \text { for all } 2 \leqslant n \leqslant m .
$$

From $F_{y}^{(2)}\left(x y^{m}\right) \neq 0$ and $\mathscr{P} f_{y}^{(-1)}\left(x y^{m}\right)$, we get $F_{y}^{(-1)}\left(x y^{m}\right)=0$. Since $f\left(x y^{m-1}\right)=f\left(x y^{m}\right)=a$ in (24), $F_{y}^{(-1)}\left(x y^{m}\right)=0$ reduces to $f\left(x y^{m+1}\right)=-2 a$. Next, we show that $m$ must be odd by contradiction. Suppose that $m=2 k$ for some $k \in \mathbb{Z}$. We have $f\left(x y^{2 k+1}\right)=f\left(x y^{m+1}\right)=-2 a$. From $f(x y)=-2 a$ in (20) and $f\left(x y^{k+1}\right)=a$ in (24), the alternatives in $\mathscr{P} f_{y^{k}}^{(-1)}\left(x y^{k+1}\right)$ give $a=0$, a contradiction. Thus $m$ must be odd. Next, we show that

$$
\begin{aligned}
& \left(f\left(x y^{m+2}\right), f\left(x y^{m+3}\right), \ldots, f\left(x y^{2 m}\right),\right. \\
& \left.f\left(x y^{2 m+1}\right)\right)=(a, a, \ldots, a,-2 a) .
\end{aligned}
$$

Let $p$ be an integer with $1 \leqslant p \leqslant m-1$. From $f\left(x y^{m-p+1}\right)=a$ in (24) and $f\left(x y^{m+1}\right)=-2 a$, the alternatives in $\mathscr{P} f_{y^{p}}^{(-1)}\left(x y^{m+1}\right)$ give $f\left(x y^{m+p+1}\right)=$ $-5 a$ or $f\left(x y^{m+p+1}\right)=a$. First, assume that $f\left(x y^{m+p+1}\right)=-5 a$. Since $0 \leqslant m-p-1 \leqslant m-2$, by (20) and (24), we have $f\left(x y^{m-p-1}\right)=-2 a$ or $f\left(x y^{m-p-1}\right)=a$. From $f\left(x y^{m}\right)=a$ in (24), the alternatives in $\mathscr{P} f_{y^{p+1}}^{(-1)}\left(x y^{m}\right)$ give $a=0$, a contradiction. Thus we must have $f\left(x y^{m+p+1}\right)=a$. Hence $f\left(x y^{n}\right)=a$ for all $m+2 \leqslant n \leqslant 2 m$. Considering the alternatives in $\mathscr{P} f_{y}^{(-1)}\left(x y^{2 m}\right)$, we get $f\left(x y^{2 m+1}\right)=a$ or $f\left(x y^{2 m+1}\right)=-2 a$. First, assume that $f\left(x y^{2 m+1}\right)=a$. From $f(x y)=f\left(x y^{m+1}\right)=$ $-2 a$, the alternatives in $\mathscr{P} f_{y^{m}}^{(-1)}\left(x y^{m+1}\right)$ give $a=0$ and therefore a contradiction. Thus we must have $f\left(x y^{2 m+1}\right)=-2 a$.

Similarly, by repeating the process of (25), we obtain

$$
\begin{aligned}
\left(f\left(x y^{2 m+2}\right), f\left(x y^{2 m+3}\right), \ldots, f\left(x y^{3 m}\right),\right. & \\
\left.f\left(x y^{3 m+1}\right)\right) & =(a, a, \ldots, a,-2 a)
\end{aligned}
$$

and so on. Eventually, we arrive at

$$
\begin{aligned}
\left(f\left(x y^{i m+2}\right),\right. & f\left(x y^{i m+3}\right), \ldots, f\left(x y^{(i+1) m}\right), \\
\left.f\left(x y^{(i+1) m+1}\right)\right) & =(a, a, \ldots, a,-2 a)
\end{aligned}
$$

for all $i \geqslant 0$. Moreover, we can similarly repeat the process of (25) for each $f\left(x y^{k}\right)$ with $k \leqslant-3$ to get $\left(f\left(x y^{n}\right)\right)_{n \in \mathbb{Z}}=(-2 a, a, \ldots, a)$, a periodic sequence of an odd period $p \geqslant 5$ as desired.

\section{MAIN RESULTS AND SOME EXAMPLES}

In this section, all lemmas in the previous section will be consolidated to provide three more lemmas, which will eventually comprise our main theorem.

We will first make the following crucial observations. Let $f \in \mathscr{A}_{(G, H)}^{(\lambda)} \backslash \mathscr{J}_{(G, H)}$ and let $x, y \in G$. By the definition of $\mathscr{A}_{(G, H)}^{(\lambda)}$ and $\mathscr{J}_{(G, H)}$, one of the following properties holds.

(i) $F_{y}^{(2)}\left(x y^{n}\right) \neq 0$ for all $n \in \mathbb{Z}$.

(ii) There exists $m \in \mathbb{Z}$ such that

(ii.1) $F_{y}^{(2)}\left(x y^{m}\right) \neq 0$ and $F_{y}^{(2)}\left(x y^{m-1}\right)=0$, or

(ii.2) $F_{y}^{(2)}\left(x y^{m}\right) \neq 0$ and $F_{y}^{(2)}\left(x y^{m+1}\right)=0$.

The above observation will be used in the proof of the following lemmas and theorem.

Lemma 5 Let $f \in \mathscr{A}_{(G, H)}^{(0)} \backslash \mathscr{J}_{(G, H)}$ and let $x, y \in G$. Then $\left(f\left(x y^{n}\right)\right)_{n \in \mathbb{Z}}=(\overline{-a}, \bar{a})$ for some $a \in H$.

Proof: Suppose that all the assumptions in the lemma hold. By the above observation, we have the following cases. 
(i) Assume that $F_{y}^{(2)}\left(x y^{n}\right) \neq 0$ for all $n \in \mathbb{Z}$. The alternatives in $\mathscr{P} f_{y}^{(0)}\left(x y^{-1}\right)$ and $\mathscr{P} f_{y}^{(0)}(x y)$ gives $F_{y}^{(0)}\left(x y^{-1}\right)=0$ and $F_{y}^{(0)}(x y)=0$, respectively. From $F_{y}^{(0)}\left(x y^{-1}\right)=0, \quad F_{y}^{(2)}(x) \neq 0$ and $F_{y}^{(0)}(x y)=0$, Lemma 3 gives a contradiction. Thus the solution does not exist in this case.

(ii) Assume that there exists $m \in \mathbb{Z}$ such that $F_{y}^{(2)}\left(x y^{m}\right) \neq 0$ and $F_{y}^{(2)}\left(x y^{m-1}\right)=0$, or $F_{y}^{(2)}\left(x y^{m}\right) \neq$ 0 and $F_{y}^{(2)}\left(x y^{m+1}\right)=0$. Hence each case implies that

(ii.1) If $F_{y}^{(2)}\left(x y^{m}\right) \neq 0$ and $F_{y}^{(2)}\left(x y^{m-1}\right)=0$, then the alternatives in $\mathscr{P} f_{y}^{(0)}\left(x y^{m}\right)$ give $F_{y}^{(0)}\left(x y^{m}\right)=0$. From $F_{y}^{(2)}\left(x y^{m-1}\right)=0$ and $F_{y}^{(2)}\left(x y^{m}\right) \neq 0$, Lemma 1 gives $F_{y}^{(2)}\left(x y^{m+1}\right) \neq 0$. We consider the alternatives in $\mathscr{P} f_{y}^{(0)}\left(x y^{m+2}\right)$ as follows. If $F_{y}^{(0)}\left(x y^{m+2}\right)=0$, then from $F_{y}^{(2)}\left(x y^{m+1}\right) \neq 0$ and $F_{y}^{(0)}\left(x y^{m}\right)=0$, we get a contradiction by Lemma 3. Thus we must have $F_{y}^{(0)}\left(x y^{m+2}\right) \neq 0$. From $F_{y}^{(2)}\left(x y^{m-1}\right)=0$, $F_{y}^{(2)}\left(x y^{m}\right) \neq 0$ and $F_{y}^{(0)}\left(x y^{m+2}\right) \neq 0$, we get

$$
f\left(x y^{m+n}\right)= \begin{cases}-f\left(x y^{m}\right), & \text { if } n \in \mathbb{Z}^{+}, \\ f\left(x y^{m}\right), & \text { if } n \in \mathbb{Z}^{-}\end{cases}
$$

by substituting $x$ by $x y^{m}$ in Lemma 2 . Hence $\left.\left(f\left(x y^{n}\right)\right)_{n \in \mathbb{Z}}=\left(\overline{f\left(x y^{m}\right)}, \overline{-f\left(x y^{m}\right.}\right)\right)$.

(ii.2) If $F_{y}^{(2)}\left(x y^{m}\right) \neq 0$ and $F_{y}^{(2)}\left(x y^{m+1}\right)=0$, then we conclude that $\left(f\left(x y^{n}\right)\right)_{n \in \mathbb{Z}}=$ $\left(\overline{-f\left(x y^{m}\right)}, \overline{f\left(x y^{m}\right)}\right)$ by replacing $x$ by $x y^{2 m}$ and $y$ by $y^{-1}$ in the arguments in the case (i.1).

Lemma 6 Let $f \in \mathscr{A}_{(G, H)}^{(-1)} \backslash \mathscr{J}_{(G, H)}$ and let $x, y \in G$. Then

(i) $\left(f\left(x y^{n}\right)\right)_{n \in \mathbb{Z}}=(\overline{a, b,-a-b})$ for some $a, b \in H$, or

(ii) $\left(f\left(x y^{n}\right)\right)_{n \in \mathbb{Z}}=(\bar{a},-2 a, \bar{a})$ for some $a \in H$, or

(iii) $\left(f\left(x y^{n}\right)\right)_{n \in \mathbb{Z}}=(\overline{-2 a, a, \ldots, a})$, a periodic sequence of an odd period $p \geqslant 5$, for some $a \in H$.

Proof: Suppose that all the assumptions in the lemma hold. By the above observation, we have the following cases.

(i) Assume that $F_{y}^{(2)}\left(x y^{n}\right) \neq 0$ for all $n \in \mathbb{Z}$. Thus the alternatives in $\mathscr{P} f_{y}^{(-1)}\left(x y^{n}\right)$ give $F_{y}^{(-1)}\left(x y^{n}\right)=$ 0, i.e., $f\left(x y^{n-1}\right)+f\left(x y^{n}\right)+f\left(x y^{n+1}\right)=0$ for all $n \in$ $\mathbb{Z}$. This implies the property (i).

(ii) Assume that there exists $m \in \mathbb{Z}$ such that $F_{y}^{(2)}\left(x y^{m}\right) \neq 0$ and $F_{y}^{(2)}\left(x y^{m-1}\right)=0$, or $F_{y}^{(2)}\left(x y^{m}\right) \neq$ 0 and $F_{y}^{(2)}\left(x y^{m+1}\right)=0$. Hence each case implies that (ii.1) Suppose $F_{y}^{(2)}\left(x y^{m}\right) \neq 0$ and $F_{y}^{(2)}\left(x y^{m-1}\right)=0$. In the case when $F_{y}^{(-1)}\left(x y^{m-1}\right) \neq 0$, we will consider the alternatives in $\mathscr{P} f_{y}^{(-1)}\left(x y^{m+2}\right)$ as follows. If $F_{y}^{(-1)}\left(x y^{m+2}\right) \neq 0$, then Lemma 2 gives a contradiction. Hence $F_{y}^{(-1)}\left(x y^{m+2}\right)=$ 0. From $F_{y}^{(-1)}\left(x y^{m-1}\right) \neq 0, F_{y}^{(2)}\left(x y^{m}\right) \neq$ 0 and $F_{y}^{(-1)}\left(x y^{m+2}\right)=0$, by replacing $x$ by $x y^{m}$ in Lemma 4, we get properties (ii) and (iii). Thus we will only consider the case when the alternatives in $\mathscr{P} f_{y}^{(-1)}\left(x y^{-1}\right)$ are equivalent, i.e., we also get $F_{y}^{(-1)}\left(x y^{m-1}\right)=$ 0 . Thus $f\left(x y^{m-1}\right)=0$ by Remark 1 . From $F_{y}^{(2)}\left(x y^{m-1}\right)=0$ and $F_{y}^{(2)}\left(x y^{m}\right) \neq 0$, we get $F_{y}^{(2)}\left(x y^{m+1}\right) \neq 0$ by Lemma 1 . Thus the alternatives in $\mathscr{P} f_{y}^{(-1)}\left(x y^{m+1}\right)$ give $F_{y}^{(-1)}\left(x y^{m+1}\right)=$ 0 . From $F_{y}^{(2)}\left(x y^{m}\right) \neq 0$ and $\mathscr{P} f_{y}^{(-1)}\left(x y^{m}\right)$, we have $F_{y}^{(-1)}\left(x y^{m}\right)=0$. Hence $f\left(x y^{m}\right) \neq 0$ by Remark 1. Let $f\left(x y^{m}\right)=a$. From $f\left(x y^{-1}\right)=$ $0, F_{y}^{(-1)}\left(x y^{m}\right)=0$ and $F_{y}^{(-1)}\left(x y^{m+1}\right)=0$, we conclude that

$$
\begin{aligned}
\left(f\left(x y^{m-1}\right), f\left(x y^{m}\right), f\left(x y^{m+1}\right),\right. \\
\left.f\left(x y^{m+2}\right)\right)=(0, a,-a, 0) .
\end{aligned}
$$

By (26), the alternatives in $\mathscr{P} f_{y}^{(-1)}\left(x y^{m+2}\right)$, $\mathscr{P} f_{y^{2}}^{(-1)}\left(x y^{m+2}\right)$ and $\mathscr{P} f_{y^{3}}^{(-1)}\left(x y^{m+2}\right)$ give

$$
\begin{array}{r}
\left(f\left(x y^{m+3}\right), f\left(x y^{m+4}\right), f\left(x y^{m+5}\right)\right) \\
=(a,-a, 0) .
\end{array}
$$

Similarly, the alternatives in $\mathscr{P} f_{y}^{(-1)}\left(x y^{m+5}\right)$, $\mathscr{P} f_{y^{2}}^{(-1)}\left(x y^{m+5}\right)$ and $\mathscr{P} f_{y^{3}}^{(-1)}\left(x y^{m+5}\right)$ give

$$
\begin{array}{r}
\left(f\left(x y^{m+6}\right), f\left(x y^{m+7}\right), f\left(x y^{m+8}\right)\right) \\
=(a,-a, 0)
\end{array}
$$

and so on. Finally, we get

$$
\begin{aligned}
& \left(f\left(x y^{m+3 k}\right), f\left(x y^{m+3 k+1}\right),\right. \\
& \left.f\left(x y^{m+3 k+2}\right)\right)=(a,-a, 0),
\end{aligned}
$$

for all $k \geqslant 0$. On the other hand, the alternatives in $\mathscr{P} f_{y}^{(-1)}\left(x y^{m-1}\right), \mathscr{P} f_{y^{2}}^{(-1)}\left(x y^{m-1}\right)$ and $\mathscr{P} f_{y^{3}}^{(-1)}\left(x y^{m-1}\right)$ give

$$
\begin{array}{r}
\left(f\left(x y^{m-2}\right), f\left(x y^{m-3}\right), f\left(x y^{m-4}\right)\right) \\
=(-a, a, 0) .
\end{array}
$$


Repeating a similar procedure to the proof of (27), we have

$$
\begin{aligned}
& \left(f\left(x y^{m-3 k}\right), f\left(x y^{m-3 k-1}\right),\right. \\
& \left.\quad f\left(x y^{m-3 k-2}\right)\right)=(a, 0,-a)
\end{aligned}
$$

for all $k \geqslant 0$. Hence we get the property (i) when $b=-a$.

(ii.2) If $F_{y}^{(2)}\left(x y^{m}\right) \neq 0$ and $F_{y}^{(2)}\left(x y^{m+1}\right)=0$, then we have similar results to the case (ii.1) by replacing $x$ by $x y^{2 m}$ and $y$ by $y^{-1}$.

Lemma 7 Let $f \in \mathscr{A}_{(G, H)}^{(-2)} \backslash \mathscr{J}_{(G, H)}$ and let $x, y \in G$. Then $f\left(x y^{n}\right)=(-1)^{n}(f(x)-n(f(x)+f(x y)))$ for all $n \in \mathbb{Z}$.

Proof: Suppose that all the assumptions in the lemma hold. Hence $F_{y}^{(2)}\left(x y^{m}\right) \neq 0$ for some $m \in \mathbb{Z}$. By the alternatives in $\mathscr{P} f_{y}^{(-2)}\left(x y^{m}\right)$, we get $F_{y}^{(-2)}\left(x y^{m}\right)=0$. Consider the alternatives in $\mathscr{P} f_{y}^{(-2)}\left(x y^{m-1}\right)$ as follows. If $F_{y}^{(-2)}\left(x y^{m-1}\right) \neq 0$, then Lemma 3 gives a contradiction. Thus we must have $F_{y}^{(-2)}\left(x y^{m-1}\right)=0$. Similarly, by Lemma 3, the alternatives in $\mathscr{P} f_{y}^{(-2)}\left(x y^{m+1}\right)$ give $F_{y}^{(-2)}\left(x y^{m+1}\right)=$ 0 . First, we will show that $F_{y}^{(-2)}\left(x y^{m+2}\right)=0$ by contradiction. Suppose $F_{y}^{(-2)}\left(x y^{m+2}\right) \neq 0$. The alternatives in $\mathscr{P} f_{y}^{(-2)}\left(x y^{m+2}\right)$ give $F_{y}^{(2)}\left(x y^{m+2}\right)=$ 0 . Hence $f\left(x y^{m+2}\right) \neq 0$ by Remark 1. From $F_{y}^{(-2)}\left(x y^{m+1}\right)=0$ and $F_{y}^{(-2)}\left(x y^{m+2}\right) \neq 0$, we get $F_{y}^{(-2)}\left(x y^{m+3}\right) \neq 0$ by Lemma 1 . Hence the alternatives in $\mathscr{P} f_{y}^{(-2)}\left(x y^{m+3}\right)$ give $F_{y}^{(2)}\left(x y^{m+3}\right)=$ 0 . Eliminating $f\left(x y^{m}\right)$ from $F_{y}^{(-2)}\left(x y^{m}\right)=0$ and $F_{y}^{(-2)}\left(x y^{m+1}\right)=0$, we get

$$
f\left(x y^{m-1}\right)-3 f\left(x y^{m+1}\right)-2 f\left(x y^{m+2}\right)=0 .
$$

Eliminating $f\left(x y^{m-1}\right)$ and $f\left(x y^{m+3}\right)$ from $F_{y}^{(2)}\left(x y^{m+2}\right)=0,(28)$ and each alternative in $\mathscr{P} f_{y^{2}}^{(-2)}\left(x y^{m+1}\right)$, we have

$$
f\left(x y^{m+2}\right)=0 \text { or } f\left(x y^{m+1}\right)+f\left(x y^{m+2}\right)=0 .
$$

On the other hand, by $F_{y}^{(2)}\left(x y^{m+2}\right)=0$ and $F_{y}^{(2)}\left(x y^{m+3}\right)=0$, we obtain

$$
2 f\left(x y^{m+1}\right)-3 f\left(x y^{m+2}\right)+f\left(x y^{m+4}\right)=0 .
$$

Eliminating $f\left(x y^{m}\right)$ and $f\left(x y^{m+4}\right)$ from $F_{y}^{(-2)}\left(x y^{m+1}\right)=0, \quad$ (30) and each alternative in $\mathscr{P} f_{y^{2}}^{(-2)}\left(x y^{m+2}\right)$, we get

$$
f\left(x y^{m+1}\right)=0 \text { or } f\left(x y^{m+1}\right)-f\left(x y^{m+2}\right)=0 .
$$

Combining (29) and (31), we conclude that $f\left(x y^{m+2}\right)=0$, a contradiction. Hence we get $F_{y}^{(-2)}\left(x y^{m+2}\right)=0$. We can repeat the above process to show $F_{y}^{(-2)}\left(x y^{m+3}\right)=0$ by replacing $x$ by $x y$ and so on. Thus we obtain

$$
F_{y}^{(-2)}\left(x y^{n}\right)=0 \text { for all } n \geqslant m+2 .
$$

Similarly, we can repeat the process of (32) for each $n \leqslant m-2$ to get $F_{y}^{(-2)}\left(x y^{n}\right)=0$ for all $n \in \mathbb{Z}$, i.e.,

$$
f\left(x y^{n+1}\right)+f\left(x y^{n}\right)=(-1)\left(f\left(x y^{n}\right)+f\left(x y^{n-1}\right)\right) .
$$

Hence $f\left(x y^{n}\right)=(-1)^{n}(f(x)-n(f(x)+f(x y)))$ for all $n \in \mathbb{Z}$.

Theorem 1 If there exists $f \in \mathscr{A}_{(G, H)}^{(\lambda)} \backslash \mathscr{J}_{(G, H)}$, then $\lambda \in\{0,-1,-2\}$. Moreover, if $x, y \in G$, then one of the following properties must hold.

(i) $\lambda=0$ and $\left(f\left(x y^{n}\right)\right)_{n \in \mathbb{Z}}=(\overline{-a}, \bar{a})$ for some $a \in$ $H \backslash\{0\}$.

(ii) $\lambda=-1$ and

(ii.1) $\left(f\left(x y^{n}\right)\right)_{n \in \mathbb{Z}}=(\overline{a, b,-a-b})$ for some $a, b \in H$ with $(a, b) \neq(0,0)$, or

(ii.2) $\left(f\left(x y^{n}\right)\right)_{n \in \mathbb{Z}}=(\bar{a},-2 a, \bar{a})$ for some $a \in$ $H \backslash\{0\}$, or

(ii.3) $\left(f\left(x y^{n}\right)\right)_{n \in \mathbb{Z}}=(\overline{-2 a, a, \ldots, a})$, a periodic sequence of an odd period $p \geqslant 5$, for some $a \in H \backslash\{0\}$.

(iii) $\lambda=-2$ and $f\left(x y^{n}\right)=(-1)^{n}(f(x)-n(f(x)+$ $f(x y))$ ) for all $n \in \mathbb{Z}$.

Proof: Let $f \in \mathscr{A}_{(G, H)}^{(\lambda)} \backslash \mathscr{J}_{(G, H)}$ and let $x, y \in G$. By the above observation, we have the following cases.

(i) Assume that $F_{y}^{(2)}\left(x y^{n}\right) \neq 0$ for all $n \in \mathbb{Z}$. The alternatives in $\mathscr{P} f_{y}^{(\lambda)}\left(x y^{-1}\right)$ and $\mathscr{P} f_{y}^{(\lambda)}(x y)$ give $F_{y}^{(\lambda)}\left(x y^{-1}\right)=0$ and $F_{y}^{(\lambda)}(x y)=0$, respectively. From $F_{y}^{(\lambda)}\left(x y^{-1}\right)=0, F_{y}^{(2)}(x) \neq 0$ and $F_{y}^{(\lambda)}(x y)=0$, Lemma 3 gives $\lambda \in\{-1,-2\}$.

(ii) Assume that there exists $m \in \mathbb{Z}$ such that $F_{y}^{(2)}\left(x y^{m}\right) \neq 0$ and $F_{y}^{(2)}\left(x y^{m-1}\right)=0$, or $F_{y}^{(2)}\left(x y^{m}\right) \neq$ 0 and $F_{y}^{(2)}\left(x y^{m+1}\right)=0$. Hence each case implies that

(ii.1) If $F_{y}^{(2)}\left(x y^{m}\right) \neq 0$ and $F_{y}^{(2)}\left(x y^{m-1}\right)=0$, then Lemma 1 gives $F_{y}^{(2)}\left(x y^{m+1}\right) \neq 0$. From $F_{y}^{(2)}\left(x y^{m}\right) \neq 0$ and $\mathscr{P} f_{y}^{(\lambda)}\left(x y^{m}\right)$, we get $F_{y}^{(\lambda)}\left(x y^{m}\right)=0$. Consider the alternatives in $\not{P} f_{y}^{(\lambda)}\left(x y^{m+2}\right)$ as follows. If $F_{y}^{(\lambda)}\left(x y^{m+2}\right) \neq 0$, then Lemma 2 gives $\lambda=0$. If $F_{y}^{(\lambda)}\left(x y^{m+2}\right)=0$, then Lemma 3 gives $\lambda=-1$ or $\lambda=-2$. 
(ii.2) If $F_{y}^{(2)}\left(x y^{m}\right) \neq 0$ and $F_{y}^{(2)}\left(x y^{m+1}\right)=0$, then we get similar results to the case (ii.1) by replacing $x$ by $x y^{2 m}$ and $y$ by $y^{-1}$ in the previous arguments.

Thus we must have $\lambda \in\{0,-1,-2\}$. According to Lemma 5, Lemma 6 and Lemma 7, we have our results corresponding to the values of the $\lambda$ 's.

Corollary 1 Let $f \in \mathscr{A}_{(G, H)}^{(\lambda)}$. If $\lambda \notin\{0,-1,-2\}$, then $f \in \mathscr{J}_{(G, H)}$.

Proof: If $\lambda \notin\{0,-1,-2\}$, then from Theorem 1 , $\mathscr{A}_{(G, H)}^{(\lambda)} \backslash \mathscr{J}_{(G, H)}$ is empty.

In other words, Corollary 1 states that when $\lambda \notin\{0,-1,-2\}$, the alternative Jensen's functional equation (3) is equivalent to Jensen's functional equation (4) for the class of functions from $(G, \cdot)$ to $(H,+)$. On the other hand, when $\lambda \in\{0,-1,-2\}$, (3) is not necessarily equivalent to (4) as illustrated in the following three examples corresponding to each value of $\lambda$.

Example 1 Given $a \in H \backslash\{0\}$. Let $f: \mathbb{R} \rightarrow H$ be a function such that

$$
f(x)= \begin{cases}-a, & x<0 \\ a, & x \geqslant 0 .\end{cases}
$$

By choosing $x=0$ and $y=1$, we have $f(x-y)-$ $2 f(x)+f(x+y)=f(-1)-2 f(0)+f(1)=-2 a$. From $a \neq 0$ and the fact that $H$ is uniquely divisible, we get $-2 a \neq 0$. Thus $f \notin \mathscr{J}_{(\mathbb{R}, H)}$. Given $x, y \in \mathbb{R}$, if $x-y \geqslant 0$ and $x+y \geqslant 0$, or $x-y<0$ and $x+y<0$, then $f(x-y)-2 f(x)+f(x+y)=0$; otherwise, $f(x-y)+f(x+y)=0$. Hence $f \in \mathscr{A}_{(\mathbb{R}, H)}^{(0)} \backslash \mathscr{J}_{(\mathbb{R}, H)}$.

Example 2 Given $a \in H \backslash\{0\}$. Let $f: \mathbb{R} \rightarrow H$ be a function such that

$$
f(x)=\left\{\begin{array}{lc}
-2 a, & x=0, \\
a, & \text { otherwise. }
\end{array}\right.
$$

By choosing $x=0$ and $y=1$, we obtain $f(x-y)-$ $2 f(x)+f(x+y)=f(-1)-2 f(0)+f(1)=6 a$. Since $a \neq 0$ and $H$ is uniquely divisible, we have $6 a \neq 0$. Thus $f \notin \mathscr{J}_{(\mathbb{R}, H)}$. Given $x, y \in \mathbb{R}$, if $(y \neq 0$ and $x-$ $y=0)$ or $(y \neq 0$ and $x=0)$ or $(y \neq 0$ and $x+y=0)$, then $f$ will satisfy $f(x-y)+f(x)+f(x+y)=0$; otherwise, we have $f(x-y)-2 f(x)+f(x+y)=0$. Thus $f \in \mathscr{A}_{(\mathbb{R}, H)}^{(-1)} \backslash \mathscr{J}_{(\mathbb{R}, H)}$.

Example 3 Given $a, b \in H$ with $a \neq-b$. Let $f: \mathbb{Z} \rightarrow$ $H$ be a function such that

$$
f(n)=(-1)^{n}(a+n b) \text { for all } n \in \mathbb{Z} .
$$

Note that $f(0)-2 f(1)+f(2)=4 a+4 b$. Since $a \neq$ $-b$ and $H$ is uniquely divisible, we have $4 a+4 b \neq$ 0 . Thus $f \notin \mathscr{J}_{(\mathbb{Z}, H)}$. Given $n, m \in \mathbb{Z}$, if $m$ is odd, then we observe that $n-m$ and $n+m$ have the same parity whereas $n$ and $n+m$ have the opposite. Hence $f(n-m)+2 f(n)+f(n+m)=0$. Otherwise, if $m$ is even, then $n-m, n, n+m$ all have the same parity. Hence $f(n-m)-2 f(n)+f(n+m)=0$. Thus $f \in$ $\mathscr{A}_{(\mathbb{Z}, H)}^{(-2)} \backslash \mathscr{J}_{(\mathbb{Z}, H)}$.

\section{GENERAL SOLUTION ON CYCLIC GROUPS}

When no additional information is provided on the group $(G, \cdot)$, Theorem 1 is probably the most general result one can obtain regarding the solution to (3). Nonetheless, for a certain group $(G, \cdot)$, one may employ Theorem 1 to obtain the general solution. In this section, we will give an application of Theorem 1 to cyclic groups. Remember that $\mathscr{J}_{(G, H)} \subseteq$ $\mathscr{A}_{(G, H)}^{(\lambda)}$. Hence Theorem 1 will help us identify the elements of $\mathscr{A}_{(G, H)}^{(\lambda)} \backslash \mathscr{J}_{(G, H)}$.

We will start with infinite cyclic groups, where Theorem 1 can be applied directly with no necessary modification as in the following theorem.

Theorem 2 Let $(G, \cdot)$ be an infinite cyclic group with $G=\langle g\rangle$. A function $f \in \mathscr{A}_{(G, H)}^{(\lambda)}$ if and only if $f \in$ $\mathscr{J}_{(G, H)}$ or one of the following properties holds.

(i) $\lambda=0$ and $\left(f\left(g^{n}\right)\right)_{n \in \mathbb{Z}}=(\overline{-a}, \bar{a})$ for some $a \in H$.

(ii) $\lambda=-1$ and

(ii.1) $\left(f\left(g^{n}\right)\right)_{n \in \mathbb{Z}}=(\overline{a, b,-a-b})$ for some $a, b \in H$, or

(ii.2) $\left(f\left(g^{n}\right)\right)_{n \in \mathbb{Z}}=(\bar{a},-2 a, \bar{a})$ for some $a \in H$, or

(ii.3) $\left(f\left(g^{n}\right)\right)_{n \in \mathbb{Z}}=(\overline{-2 a, a, \ldots, a})$, a periodic sequence of an odd period $p \geqslant 5$, for some $a \in H$.

(iii) $\lambda=-2$ and $f\left(g^{n}\right)=(-1)^{n}(a+n b)$ for all $n \in \mathbb{Z}$ and for some $a, b \in H$.

Proof: Let $f \in \mathscr{A}_{(G, H)}^{(\lambda)}$. If $f \notin \mathscr{J}_{(G, H)}$, then setting $x=e$ and $y=g$ in Theorem 1, we can see that one of the properties (i), (ii), and (iii) must hold. The converse can be directly verified.

It now remains to apply Theorem 1 to finite cyclic groups, where some modification must be made to eliminate redundant possibilities. It should be remarked that for the trivial group of order 1 , we obtain a trivial result that any function $f: G \rightarrow H$ will satisfy both (3) and (4). For finite cyclic groups of order at least 2 , we have the following theorem.

Theorem 3 Let $(G, \cdot)$ be a finite cyclic group with $G=$ $\langle g\rangle$ and let $m \geqslant 2$ be an order of $G$. A function $f \in$ 
$\mathscr{A}_{(G, H)}^{(\lambda)}$ if and only if $f \in \mathscr{J}_{(G, H)}$ or one of the following properties holds.

(i) $\lambda=-1$ and

(i.1) $3 \mid m$ and $\left(f\left(g^{n}\right)\right)_{n \in \mathbb{Z}}=(\overline{a, b,-a-b})$ for some $a, b \in H$, or

(i.2) $\left(f\left(g^{n}\right)\right)_{n \in \mathbb{Z}}=(\overline{-2 a, a, \ldots, a})$, a periodic sequence of an odd period $p \geqslant 5$ with $p \mid m$, for some $a \in H$.

(iii) $\lambda=-2, m$ is even and $f\left(g^{n}\right)=(-1)^{n}$ a for all $n \in \mathbb{Z}$ and for some $a \in H$.

Proof: Given one of the above properties, it can be directly verified that $f \in \mathscr{A}_{(G, H)}^{(\lambda)}$. Conversely, let $f \in$ $\mathscr{A}_{(G, H)}^{(\lambda)}$. If $f \notin \mathscr{J}_{(G, H)}$, then setting $x=e$ and $y=g$ in Theorem 1, we have the following possibilities.

(i) $\lambda=0$ and $\left(f\left(g^{n}\right)\right)_{n \in \mathbb{Z}}=(\overline{-a}, \bar{a})$ for some $a \in H$.

(ii) $\lambda=-1$ and

(ii.1) $\left(f\left(g^{n}\right)\right)_{n \in \mathbb{Z}}=(\overline{a, b,-a-b})$ for some $a, b \in H$, or

(ii.2) $\left(f\left(g^{n}\right)\right)_{n \in \mathbb{Z}}=(\bar{a},-2 a, \bar{a})$ for some $a \in H$, or

(ii.3) $\left(f\left(g^{n}\right)\right)_{n \in \mathbb{Z}}=(\overline{-2 a, a, \ldots, a})$, a periodic sequence of an odd period $p \geqslant 5$, for some $a \in H$.

(i) $\lambda=-2$ and $f\left(g^{n}\right)=(-1)^{n}(a+n b)$ for all $n \in \mathbb{Z}$ and for some $a, b \in H$.

However, not all of the above possibilities are admissible when $G$ is a finite cyclic group. We will treat each case separately and deem case (i) and case (ii.2) redundant, while the other cases are admissible with some additional conditions.

(i) $\left(f\left(g^{n}\right)\right)_{n \in \mathbb{Z}}=(\overline{-a}, \bar{a})$ for some $a \in H$, that is, there exists $k \in \mathbb{Z}$ such that

$$
f\left(g^{n}\right)= \begin{cases}-a, & n<k, \\ a, & n \geqslant k .\end{cases}
$$

Hence $f\left(g^{k-1}\right)=-a$ and $f\left(g^{k+m-1}\right)=a$. Since $m$ is the order of the group $G$, we have $g^{k-1}=g^{k+m-1}$. Thus $a=-a$, which implies that $a=0$. Hence $f \in$ $\mathscr{J}_{(G, H)}$ which deems this case redundant.

(ii) $\lambda=-1$.

(ii.1) $\left(f\left(g^{n}\right)\right)_{n \in \mathbb{Z}}=(\overline{a, b,-a-b})$ for some $a, b \in$ $H$. Suppose that $3 \nmid m$. Then $\{0, m, 2 m\}$ is a complete residue modulo 3 . Therefore

$$
\left\{f(e), f\left(g^{m}\right), f\left(g^{2 m}\right)\right\}=\{a, b,-a-b\} .
$$

But $m$ is the order of $G$, thus $g^{2 m}=g^{m}=e$. Hence $a=b=-a-b$, which gives $a=b=0$ and, in turn, $f \in \mathscr{J}_{(G, H)}$. Hence $3 \mid \mathrm{m}$.

(ii.2) $\left(f\left(g^{n}\right)\right)_{n \in \mathbb{Z}}=(\bar{a},-2 a, \bar{a})$ for some $a \in H$, that is, there exists $k \in \mathbb{Z}$ such that

$$
f\left(g^{n}\right)=\left\{\begin{array}{lc}
-2 a, & n=k, \\
a, & \text { otherwise }
\end{array}\right.
$$

Hence $f\left(g^{k}\right)=-2 a$ and $f\left(g^{k+m}\right)=a$. Since $m$ is the order of the group $G$, we have $g^{k}=g^{k+m}$. Thus $-2 a=a$, which implies that $a=0$. Hence $f \in \mathscr{J}_{(G, H)}$ which deems this case redundant.

(ii.3) $\left(f\left(g^{n}\right)\right)_{n \in \mathbb{Z}}=(\overline{-2 a, a, \ldots, a})$, a periodic sequence of an odd period $p \geqslant 5$, for some $a \in H$, that is, there exists $k \in \mathbb{Z}$ such that

$$
f\left(g^{k}\right)=-2 a
$$

Suppose that $p \nmid m$. Since $\left(f\left(g^{n}\right)\right)_{n \in \mathbb{Z}}$ is a periodic sequence of a period $p$, we must have $f\left(g^{k+m}\right)=a$. But $m$ is the order of $G$, thus $g^{k+m}=g^{k}$. Hence $-2 a=a$, which gives $a=0$, and, in turn, $f \in \mathscr{J}_{(G, H)}$. Hence $p \mid m$.

(i) $f\left(g^{n}\right)=(-1)^{n}(a+n b)$ for all $n \in \mathbb{Z}$ and for some $a, b \in H$. Since $e=g^{m}=g^{2 m}$, we have

$$
a=(-1)^{m}(a+m b)=(-1)^{2 m}(a+2 m b)
$$

which implies that $b=0$ and $m$ is even.

\section{REFERENCES}

1. Kannappan PL, Kuczma M (1974) On a functional equation related to the Cauchy equation. Ann Polon Math 30, 49-55.

2. Ger R (1977) On an alternative functional equation. Aequationes Math 15, 145-62.

3. Kuczma M (1978) On some alternative functional equations. Aequationes Math 2, 182-98.

4. Forti GL (1979) La soluzione generale dell'equazione funzionale $\{c f(x+y)-a f(x)-b f(y)-d\}\{f(x+y)-$ $f(x)-f(y)\}=0$. Le Matematiche 34, 219-42.

5. Le C-T, Thai T-H (2011) Jensen's functional equation on the symmetric group $S_{n}$. Aequationes Math 82, 269-76.

6. Ng CT (1999) Jensen's functional equation on groups, II. Aequationes Math 58, 311-20.

7. Ng CT (2001) Jensen's functional equation on groups, III. Aequationes Math 62, 143-59.

8. $\mathrm{Ng}$ CT (2005) A Pexider-Jensen functional equation on groups. Aequationes Math 70, 131-53.

9. Stetkær H (2003) On Jensen's functional equation on groups. Aequationes Math 66, 100-18.

10. Nakmahachalasint P (2012) An alternative Jensen's functional equation on semigroups. Sci Asia 38, 408-13.

11. Ng CT (1990) Jensen's functional equation on groups. Aequationes Math 39, 85-99.

12. Parnami JC, Vasudeva HL (1992) On Jensen's functional equation. Aequationes Math 43, 211-8. 